\title{
COMPUTER AIDED MECHANOGENESIS OF SKELETAL MUSCLE ORGANS FROM SINGLE CELLS IN VITRO
}

Herman H. Vandenburgh", Somporn Swasdison* \& Patricia Karlisch*

"Department of Pathology, Brown University and The Miriam Hospital, Providence, RI 02906, USA

*Department of Cell Biology and Anatomy, University of Alabama, Birmingham, AL 35294, USA

Complex mechanical forces generated in the growing embryo play an important role in organogenesis. Computerized application of similar forces to differentiating skeletal muscle myoblasts in vitro generate three dimensional artificial muscle organs. These organs contain parallel networks of long unbranched myofibers organized into fascicle-like structures. Tendon development is initiated and the muscles are capable of performing directed, functional work. Kinetically engineered organs provide a new method for studying the growth and development of normal and diseased skeletal muscle.

\section{Correspondence to:}

Dr. H. H. Vandenburgh

Department of Pathology

The Miriam Hospital

164 Summit Avenue

Providence, RI 02906

Tel. No. 401-331-8500, Ext. 4273

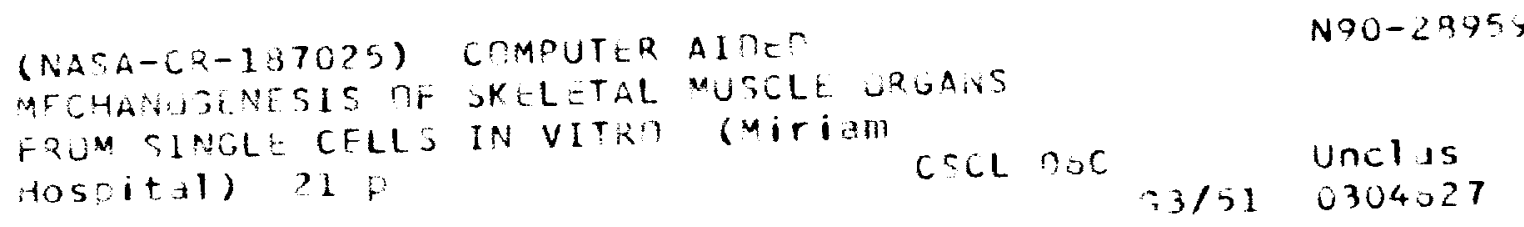


Differentiation and maintenance of specific cell phenotypes in vitro require supplying the proper extracellular matrix, nutrients, and growth factors. Even under optimal culture conditions, extensive intercellular interactions rarely occur which lead to the organization of the cells into three dimensional organs comparable to their in vivo counterpart. Spatial and chemical gradients play important roles in early organization of embryonic tissues $(1,2)$ while mechanical forces generated in the enlarging embryo by the growing skeleton, contracting muscles, and fluid movements stimulate subsequent organogenesis (3). These mechanical forces, missing in normal static cell cultures, play important growth regulatory roles in bone (4), cartilage (5), connective tissue (6), endothelial tissue (7), epithelial tissue (8), lung (9), cardiac muscle (10), smooth muscle (11), skeletal muscle (12), and nerve ( 13$)$

organogenesis occurs in some monolayer cultures when growth conditions allow the development of internally generated mechanical forces (14-16). The development of computerized mechanical cell stimulators for tissue cultured cells allows the additional application of in vivo-like external forces to monolayer cultures $(17,18)$. In this report complex mechanical forces which simulate embryonic skeletal growth and the repetitive tensions generated in actively contracting muscles are applied to monolayers of mononucleated embryonic avian skeletal muscle cells. These externally applied forces induce muscle organogenesis and lead to 
the formation of in vivo-like three dimensional functional organs. Applying mechanical forces to tissue cultured cells is another step toward complete simulation of the in vivo environment and may induce organogenesis of functional organs from many different cell types for in vitro study or in vivo transplantation.

Embryonic mononucleated avian pectoralis muscle cells are isolated by standard culture techniques and grown attached to a collagen-coated elastic substratum (17). Under static culture conditions these cells undergo the first stages of myogenesis (19). The myoblasts proliferate and fuse into multinucleated myotubes which become striated and contractile. The myotubes which form are an unorganized network of short, branching cells incapable of performing functional i.e. directed work. During the same developmental period in vivo, myotubes organize into parallel arrays of long, unbranched fibers grouped into small mosaic fascicles capable of generating tension via the tendons against the attached bones (20). The passive and active mechanical forces generated in vivo by the rapidly enlarging skeleton and by active muscle contractions, respectively, help to regulate muscle growth and organization in vivo $(12,21)$ but are missing in static cel1 cultures.

The proliferating mononucleated muscle cells growing on the elastic substratum in vitro are mechanically stimulated over a three to four week period by varying activity patterns (Figure 1). The cell monolayer is stretched and relaxed in a horizontal mechanical cell stimulator by holding one end of each elastic 
culture well stationary while the other end moves in $33 \mu \mathrm{m}$ step increments (18). A direct relationship exists between the percent of substratum stretch and the percent of cell stretch in this model system $(22,23)$. Initial substratum movement (18-54 $\mathrm{h}$ postplating) is a unidirectional stretching pattern (Fig. 1, Phase I) to simulate the rapidly elongating bone during early embryogenesis. The rate of movement $(0.33 \mathrm{~mm} / \mathrm{h})$ is comparable to the most rapid rate of limb elongation in the chick embryo [Stages 18-30] (24). This unidirectional substratum stretching stimulates cell proliferation, myoblast fusion, and orients the developing myotubes in the direction of stretch $(17,25)$. BY Day 5 postplating the oriented myotubes are 2 to 4 times longer (1-4 mm) than unoriented myotubes grown under static culture conditions $(0.5-1.0 \mathrm{~mm})$ while myotube diameters are similar in the two groups (18). Rates of unidirectional substratum stretching faster or slower than 0.14 to $0.33 \mathrm{~mm} / \mathrm{h}$ do not orient the cells as well (25). Also, repetitive stretch/relaxation of the substratum during this pericd of myoblast proliferation and fusion orients the developing myotubes perpendicular to the direction of movement rather than parallel to it $(18)$.

The monolayer of oriented myotubes is maintained in a quiescent state for several days (Fig. 1, Phase II). During this period, the myotubes develop the structural strength to withstand subsequent intense repetitive stimulations for several weeks without nyofiber damage $(26,27)$. Repetitive stretch/relaxation of the cultured myotubes soon after their formation without this rest 
period results in irreversible damage to the cells $(26,27)$. Quiescent growth periods also exist during embryonic growth in vivo although not for such an extended period (28). The process of myotube formation in vitro is much more rapid than in vivo (29) and myotubes in vivo have more time to develop an extensive extracellular matrix coat before rigorous repetitive mechanical forces begin (20). In initial experiments, the myotubes were embedded in a three-dimensional collagen matrix during this rest period to stimulate the formation of an extracellular matrix around the individual myotubes $(30,31)$. In the experiments reported here, the muscle cultures are grown at high cell densities (2.9-4.7 $\mathrm{x}$ $10 \% / \mathrm{mm}^{2}$ ) without exogenous collagen. These cultures contain many fibroblasts which are essential for the synthesis and organization of the extracellular matrix (30) and are adequate for preparing the developing myofibers for mechanical stimulation.

Repetitive stimulation initiated on Day 7-8 postplating consists of five $5 \%$ to $20 \%$ substratum stretches/relaxations during a 20 sec period, followed by a $10 \mathrm{sec}$ rest; this pattern repeats three times, followed by a 5 to $30 \mathrm{~min}$ rest period (Figure 1, Phase III insert). At the same time unidirectional substratum stretching is also initiated $(0.25-1.0 \mathrm{~mm} / 24 \mathrm{~h})$. These stimulation patterns continue for two to three weeks and partially simulate the complex perinatal muscle movements initiated in vivo as the skeleton continues to enlarge at a slower rate $(24,32)$. The repetitive stretching pattern begins as a low intensity (5\% stretch/relaxations) and infrequent (30 min rests) stimulus, and 
its intensity and frequency increase with time in culture to $20 \%$ stretch/relaxations with 5 min rest periods by the third week in culture. Unidirectional substratum stretching continues throughout this period. After six to ten days of this activity, the monolayer of cells detaches from the elastic substratum along its entire length and is held in place, under tension, at its ends by stainless steel wire mesh incorporated into each rectangular culture well at the start of an experiment. The proliferating cells grow and attach to these support structures during the first week of culture. The tissue is thus floating in the culture medium by two weeks of culture. Over the next 7 to 14 days the outer longitudinal edges of the monolayer roll upward and inward as mechanical stimulation continues, integrating into the central portion of the monolayer to form a solid rod-like structure. The tissue is always under tension since it rapidly retracts from an initial length of 30 to $35 \mathrm{~mm}$ to 20 to $25 \mathrm{~mm}$ when one end is clit free of the wire mesh.

Three to four weeks of repetitive mechanical activity results in muscle organs approximately $30-35 \mathrm{~mm}$ in length and 1-2 $\mathrm{mm}$ in diameter (Fig. 2). Muscles can be kinetically generated to varied lengths with different stimulation patterns. The ends of the muscle can also be modified in shape by changing the geometry of the attachment wire mesh (unpublished observations). Unstretched control cultures 3 to 4 weeks of age have typically unorganized, branching myofibers (Fig. 3A) averaging $0.5 \mathrm{~mm}$ to $1.0 \mathrm{~mm}$ in length. In contrast, mechanically stimulated cultures contain parallel 
arrays of long unbranched cells (Fig. 3B). By 3 to 4 weeks of culture, many of the organized myofibers are greater than 10-15 mm in length. Visualized in cross section, the folded monolayer has integrated into a solid tissue with a well defined epimysium made up of mononucleated fibroblasts (Figure 4A, arrow). Fibroblasts play an active role in organizing premuscle masses into individual muscles in vivo $(33,34)$, and in vitro stream from the epimysium into the muscle tissue, dividing it into fascicle-like groups of muscle fibers (Fig. $4 \mathrm{~A}$ and $\mathrm{B}$ ). The muscle cells in cross section begin to display a polygonal checkerboard pattern similar to that seen in vivo, with both primary and secondary-like myofibers (Fig. 4B, arrow). The myofibrillar proteins are well organized within the myofibers (Figure 4C). The tissue thus displays many characteristics of a newly developed in vivo muscle.

Nonmuscle mesenchymal cells help guide muscle tendon development in vivo, laying down the proper extracellular matrix components by which the muscle fibers attach to the skeletal system (33-37). Collagen, fibronectin, and laminin evenly distribute throughout the length of the developing muscle's extracellular matrix whereas the myotendinous antigen, tenascin, concentrates in the extracellular matrix at the myotendinous junction $(38,39)$.

Interstitial fibroblasts synthesize the majority of tenascin in muscle tissue and tenascin serves as a good marker for myotendinous junction development $(38,39)$. In static muscle cultures, both fibronectin and tenascin have a random distribution (38, 39). static 20 to 28 -day-old control cultures grown in the mechanical 
cell stimulator culture wells also stain uniformily throughout the muscle length for both fibronectin and tenascin (data not shown). Twenty to twenty-eight-day kinetically generated muscles stain uniformly for fibronectin but tenascin staining concentrates at the stationary end of the muscle (Fig. 5A). In cross section, the extracellular matrix surrounding the myofibers at the stationary end is much thicker and more intensely stained with tenascin antibody than the middle or moving end of the muscle (Figure $5 \mathrm{C}$ and D). The end of the muscle which stains most intensely with tenascin is also the end in which the cells are under the greatest tension in the mechanical cell stimulator (18). Mechanical stimulation of the cultured muscle cells is thus able to initiate myotendinous development in vitro, based on tenascin localization, and mechanical force may be one of the environmental signals in the embryo which helps to initiate this process.

Continuous repetitive mechanical stimulation of the muscle organs results in an increase in the amount of work performed by the cells, based on lactate production measured with a YSI Model 2000 Glucose/Lactate Analyzer. In a typical experiment, cells mechanically stimulated from Days 16 to 24 postplating have a mean ( \pm S.D.) lactate efflux of $1.40 \pm 0.37 \mathrm{~g} / \mathrm{mg}$ noncollagenous protein/day while static control cells produce only $0.71 \pm 0.24$ $\mathrm{g} / \mathrm{mg}$ noncollagenous protein/day of lactate $(\mathrm{P}<.001)$. Conversely, we determined whether these muscles could perform functional work by generating increased tension in the longitudinal axis of the organ in response to an external stimulus. Resting tension in four 
individual 20-26 day old muscles is $149 \pm 47$ mdynes/muscle at $4^{\circ} \mathrm{C}$ (40). Elevation of extracellular potassium to $75 \mathrm{mM}$ induces rapid contraction of the muscles and increases longitudinal axial tension to $285 \pm 53$ mdynes/muscle within 30-60 seconds. This represents a mean increase of $91 \%$ in tension development in the muscles ( $P<.001$ ) and these artificial organs can thus perform directed, functional work in response to an external stimulus.

Kinetically generated skeletal muscles provide a new model system for studying the basic processes by which mechanical forces help to regulate skeletal muscle organogenesis and growth. They also may be useful for analyzing human skeletal muscle disorders in vitro. Under normal static monolayer culture conditions human muscle cells maintain their embryonic characteristics and often the diseased muscle cells do not express the phenotype of their disorder (41). With the more complete organogenic development induced when muscle cells undergo in vivo-like mechanical stimulation, phenotypic expression of the humar muscle disorder is more likely to occur in vitro. Mechanical stimulation of cultured human skeletal muscle cells may be of particular interest in the study of Duchenne muscular dystrophy where the defective gene product dystrophin may be a structural support protein $(42,43)$ or regulator of stretch sensitive ionic channels (44). 


\section{REFERENCES}

1. Summerbell, D., Lewis, J., \& Wolpert, L. Nature 244, 492-496 $(1973)$

2. Summerbe11, D. J. Embryol. Exp. Morph. 32, 227-237 (1974).

3. Weiss, P. Am. Naturalist 67, 389-407 (1933).

4. Dewitt, M.T., Handley, C.J., Oakes, B.W. \& Lowther, D.A. Connect. Tiss. Res. 12, 97-109 (1984).

5. Klein-Nulend, J. et al. J. Biol. Chem. 262, 15490-15495 (1987).

6. Curtis, A.G.S. \& Sehar, G.M. Nature (Lond.) 274, 52-53 (1978).

7. Kamiya, A. \& Togawa, T. Am. J. Physio1. 239, H14-H21 (1980).

8. Odell, G.M., Oster, G., Alberch, P.\& Burnside, B. Dev. Biol. 85, 446-462(1981).

9. Rannels, D.E. Am. J. Physiol. 257, L179-L189 (1989).

10. Morgan, H.E. et al. Am. Rev. Physiol. 49, 533-543 (1987).

11. Leung, D.Y.M., Glagov, S., \& Mathews, M.B. Science 191, 475477 (1976).

12. Stewart, D. in Regulation of Organ and Tissue Growth (ed. R.J. Goss) 77-100 (Academic Press, New York, 1972).

13. Bray, D. Dev. Biol, 102, 379-389 (1984).

14. Folkman, J. \& Haudenschild, C. Nature 288, 551-556 (1980).

15. Ingber, D.E. \& Folkman, J. Cel1 58, 803-805 (1989).

16. Strohman, R.C., Bayne, E., Spector, D., Obinata, T.,MicouEastwood, J. \& Maniotis, A. In Vitro 26, 201-208 (1990).

17. Vandenburgh, H.H. In Vitro 24, 609-619 (1988). 
18. Vandenburgh, H.H. \& Karlisch, P. In Vitro 25, 607-616 (1989).

19. Pearson, M.L. and Epstein, H.F. (Eds.), Muscle Development: Molecular and cellular control (Cold spring Harbor, New York, $1982)$.

20. Kelly, A.M. in Handbook of Physiology. Section 10: Skeletal Muscle (Eds. Peachey, L.D., Adrian, R.H. \& Geiger, S.R.) 507538 (Am. Physiol. Soc., Bethesda, 1983).

21. Tomanek, R. Dev. Biol, 42, 305-314 (1975).

22. Vandenburgh, H.H. \& Kaufman, S. Science 203, 265-268 (1979). 23.. Vandenburgh, H.H. Med. Science Sports Med. 19, S142-S146 $(1987)$

24. Ede, D.A. in The cell surface in Animal Embryogenesis and Development (Eds. Poste, G., \& Nicolson, G.L.) 495-543 (Elsevier/North Holland, Amsterdam, 1976).

25. Vandenburgh, H.H. Dev. Biol. 93, 438-443 (1982).

26. Hatfaludy, S., Shansky, J., \& Vandenburgh, H.H. Am. J. Physiol. 256, C175-C181 (1989).

27. Vandenburgh, H.H., Hatfaludy, S., Karlisch, P. \& Shansky, J. Am. J. Physiol. 256, C674-C682 (1989).

28. Hall, B.K. clin. Orthop. 74, 249-268 (1971).

29. Bischoff, R. in Membrane Fusion (Eds. Post, G. and Nicolson, A.L.) 127-179 (Elsevier/North Holland, Amsterdam, 1978).

30. Sanderson, R.D., Fitch, J.M., Linsemayer, T.R., \& Mayne, R. I. Cell Biol. 102, 740-747 (1986).

31. Vandenburgh, H.H., Karlisch, P., \& Farr, L. In Vitro 24, 166$174(1988)$. 
32. Landmesser, L.T. \& O'Donovan, M.J. I. Physiol. 347, 189-204 (1984).

33. Chiquet, M., Eppenberger, H.M., \& Turner, D.C. Dev. Biol. 88, $220-235(1981)$.

34. Shellswell, G.B. \& Wolpert, L. in Vertebrate Limb and Somite Morphogenesis (Eds. Ede, D.A., Hinchcliffe, J.R. \& Ball, M.) 71-86 (Cambridge Univ. Press, London, 1977).

35. Shellswell, G.B., Bailey, A.J., Duance, V.C. \& Restall, D.J. J. Embryol. Exp. Morphol. 60, 245-254 (1980).

36. Kieny, M. \& Chevallier, A. J. Embryol. Exp. Morphol. 49, 153$164(1979)$.

37. Von der Mark, K., Von der Mark, H. \& Gay, S. Dev. Biol. 53, $153-170(1976)$.

38. Chiquet, M. \& Fambrough, D.M. J. Cell Biol. 98, 1926-1936 $(1984)$.

39. Chiquet, M. \& Fambrough, D.M. J. Cell Biol. 98, 1937-1946 $(1984)$.

40. Dennerll, T.J., Joshi, H.C., Steel, V.L., Buxbaum, R.E., \& Heidemann, S.R. J. Cell Biol. 107, 665-674 (1988).

41. Miranda, A.F., Mongini, J. \& DiMauro, S. Adv. Cell Culture 4, $1-45(1985)$.

42. Zubryzcka-Garn, E.E. et al. Nature 333, 466-469 (1988).

43. Watkins, S.C., Hoffman, E.P., Slayter, H.S. \& Kunkel, L.M. Nature $333,863-866$ (1988).

44. Franco, A., Jr. \& Lansman, J.B. Nature 344, 670-673 (1990). 


\section{ACKNOWLEDGEMENTS.}

Supported by NIH Grants to H.H.V. and R. Mayne, a NASA Grant to H.H.V., and a Muscular Dystrophy Association Grant to R. Mayne. Dr. E. Bandman provided embryonic avian myosin heavy chain antibody EB165. Fibronectin antibody B3/D6 (D. Fambrough) and tenascin antibody M1/B4 (D. Fambrough) were obtained from the Developmental Studies Hybridoma Bank, Baltimore, MD under an NIH Contract. We thank $X$. Xiong for technical assistance, M. Sheff, N.Fausto, and R. Goss for critical reading of the manuscript, and $D$. Leach for manuscript preparation. 
FIGURE LEGENDS

Eigure 1. Mechanical activity patterns which stimulate skeletal muscle organogenesis in tissue culture. Avian skeletal muscle cells are stretched and relaxed while differentiating on an elastic substratum (silastic ${ }^{m}$ ). Mechanical activity is regulated by a computer controlled stepper motor attached to the elastic culture wells (18). Phase I - $33 \mu \mathrm{m}$ unidirectional stretch every 6 min for 36-40 $\mathrm{h}$, representing rapid skeletal growth and the resultant stretching of the attached muscle mass. In vitro, this pattern results in myotube orientation and longitudinal growth in the direction of movement. Phase II - A quiescent mechanical period in which extracellular matrix components strengthen the myotubes. Phase III - Repetitive stretch/relaxation of the substratum combined with unidirectional stretching, representing the start of active embryonic movements together with continued enlargement of the embryo at a slower rate. Upward spikes graphically represent repetitive stretches and relaxations (see insert) of the tissue and do not represent frequency or intensity of actual stimulation. See text for details.

Figure 2. Artificial skeletal muscle organs generated by mechanical forces. Twenty-four day postplating muscle organs in elastic culture wells. Tissue is dark brown from immunocytochemical staining for different muscle proteins. The muscles are floating in the medium, attached at each end to stainless steel wire mesh. 
Upper aluminum bar remains stationary in the mechanical cell stimulator while lower bar is stretched and relaxed in the horizontal plane by the pattern in Figure 1. Arrows indicate direction of movement and bar is $8 \mathrm{~mm}$.

Eigure 3. orientation and growth of skeletal myofibers with mechanical stimulation. Higher magnification of the ends of unstretched static control muscles (A) and mechanically stimulated muscles (B) from Figure 2. Myotubes are stained with a monoclonal antibody against embryonic avian myosin heavy chain (EB165). Bar represents $0.5 \mathrm{~mm}$.

Figure 4. organization of myofibers and myofibrillar proteins within the artificial muscle organs. ( $A$ and $B$ ) Toluidine blue stained cross sections of the central portion of a 28 day postplating muscle. One-half of the muscle is shown in (A) with a well developed epimysium (arrow) and groups of darkly stained peripheral myofibers and lightly stained interior myofibers. Fibroblasts are dividing the myofibers into groupings (white arrow). Bar represents $120 \mu \mathrm{m}$. At higher magnification (B) the mosaics of myotubes with primary and secondary-like myofibers (arrow) are seen. Bar represents $15 \mu \mathrm{m}$. (C) Electron micrograph of portions of two muscle fibers showing a well organized basal lamina, caveolae, T-tubules, and myofibrillar proteins. Bar represents $2.0 \mu \mathrm{m}$. 
Eigure 5. Initiation of tendon development in kinetically generated muscles. (A) whole muscles 24-28 days postplating immunocytochemically stained with monoclonal antibodies for either fibronectin (upper muscle), or the myotendinous antigen tenascin (lower muscle). Double headed arrow indicates direction of mechanical stimulation. The left end of each muscle was attached to the moving aluminum bar while the right end was held stationary. Fibronectin staining is uniform from one end of the muscle to the other while tenascin staining. is greater at stationary end of muscle (arrowhead). (B - D) Cryostat sections immunocytochemically stained for embryonic myosin heavy chain (B), or tenascin at the moving end (C) or stationary end (D) of the muscle. Note greater thickness and more intense staining of endomysium in (D) than in (C). Bar represents $5.0 \mathrm{~mm}$ in A. 


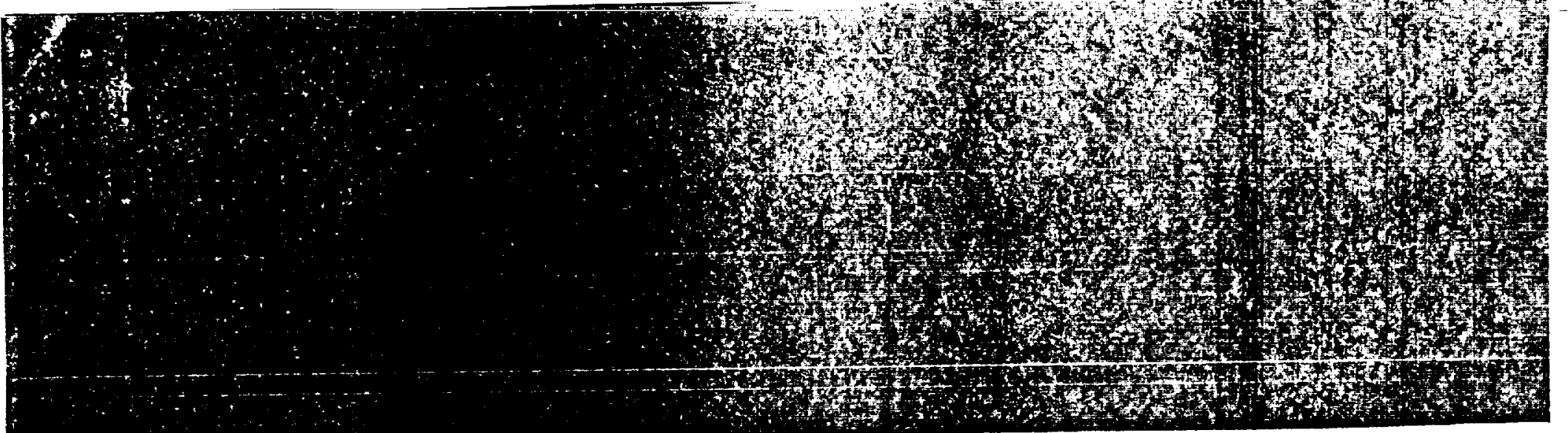

\section{FIGURE 2}
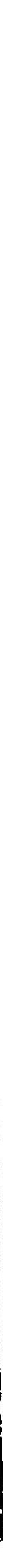


\section{FIGURE 4}
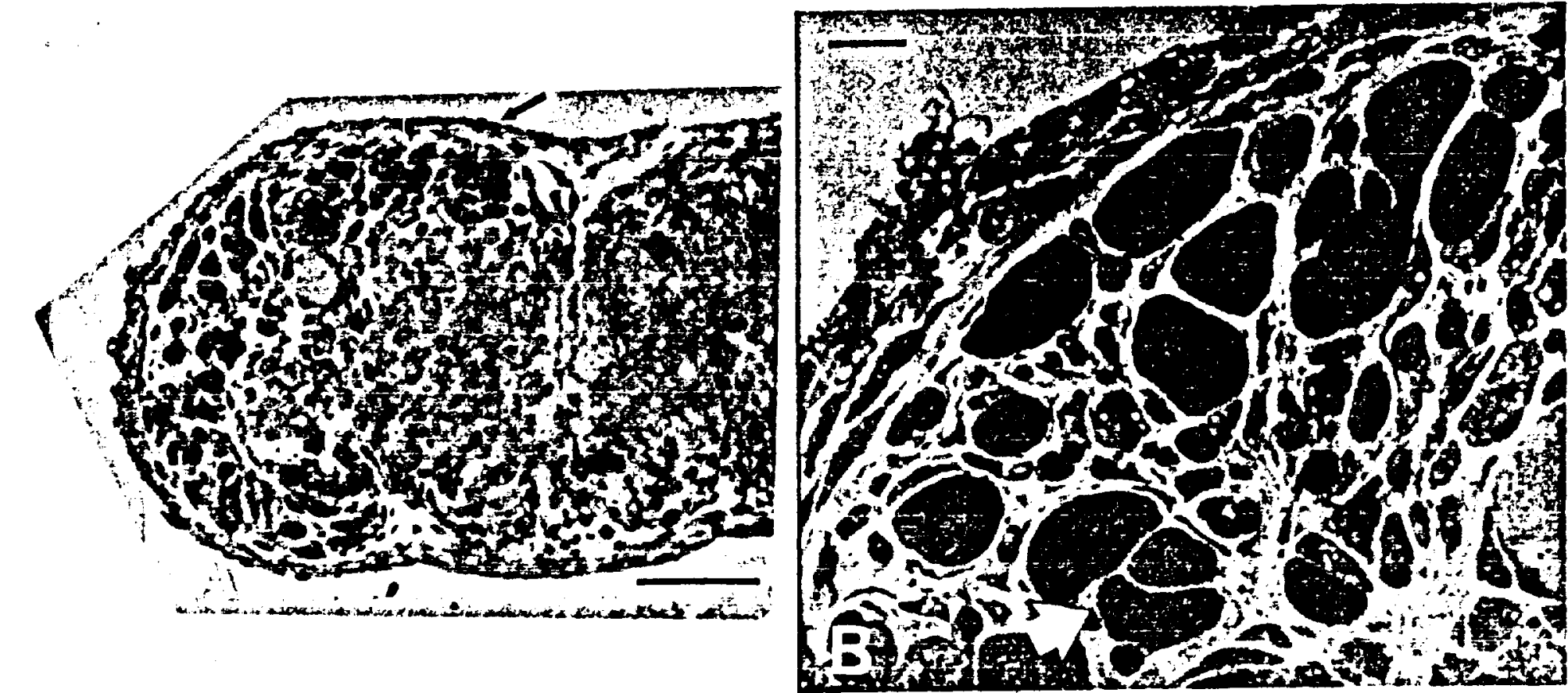

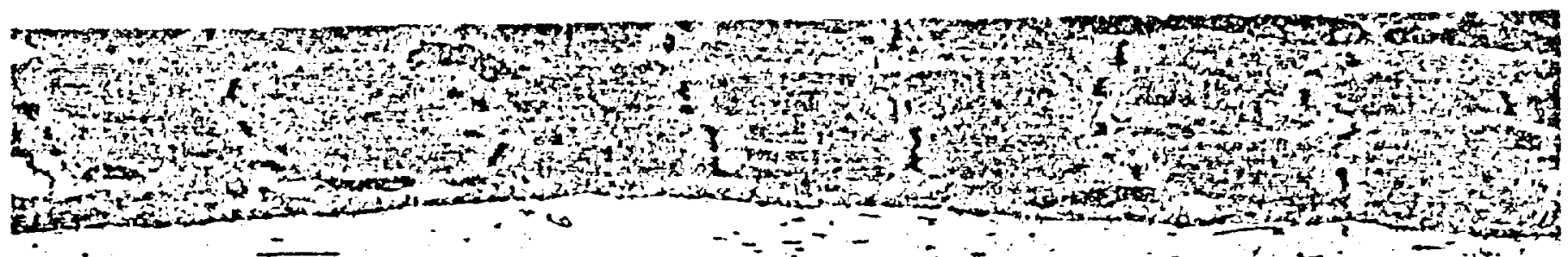

Rom

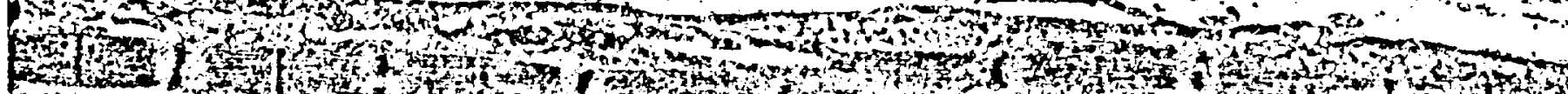

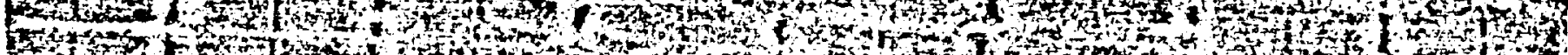
170 f

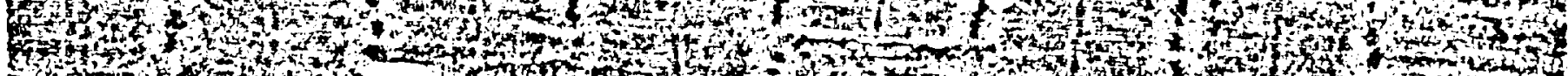

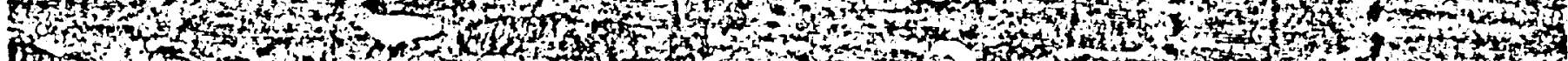
The

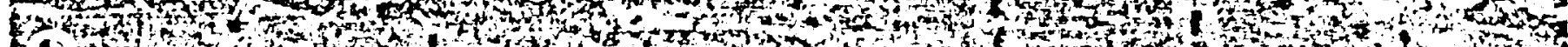
2 (1) or w

Vandenburgh et al. "Computer Aided Mechanogenesis of Skeletal Muscle organs from single Celis In Vitro" 


\section{FIGURE 5}
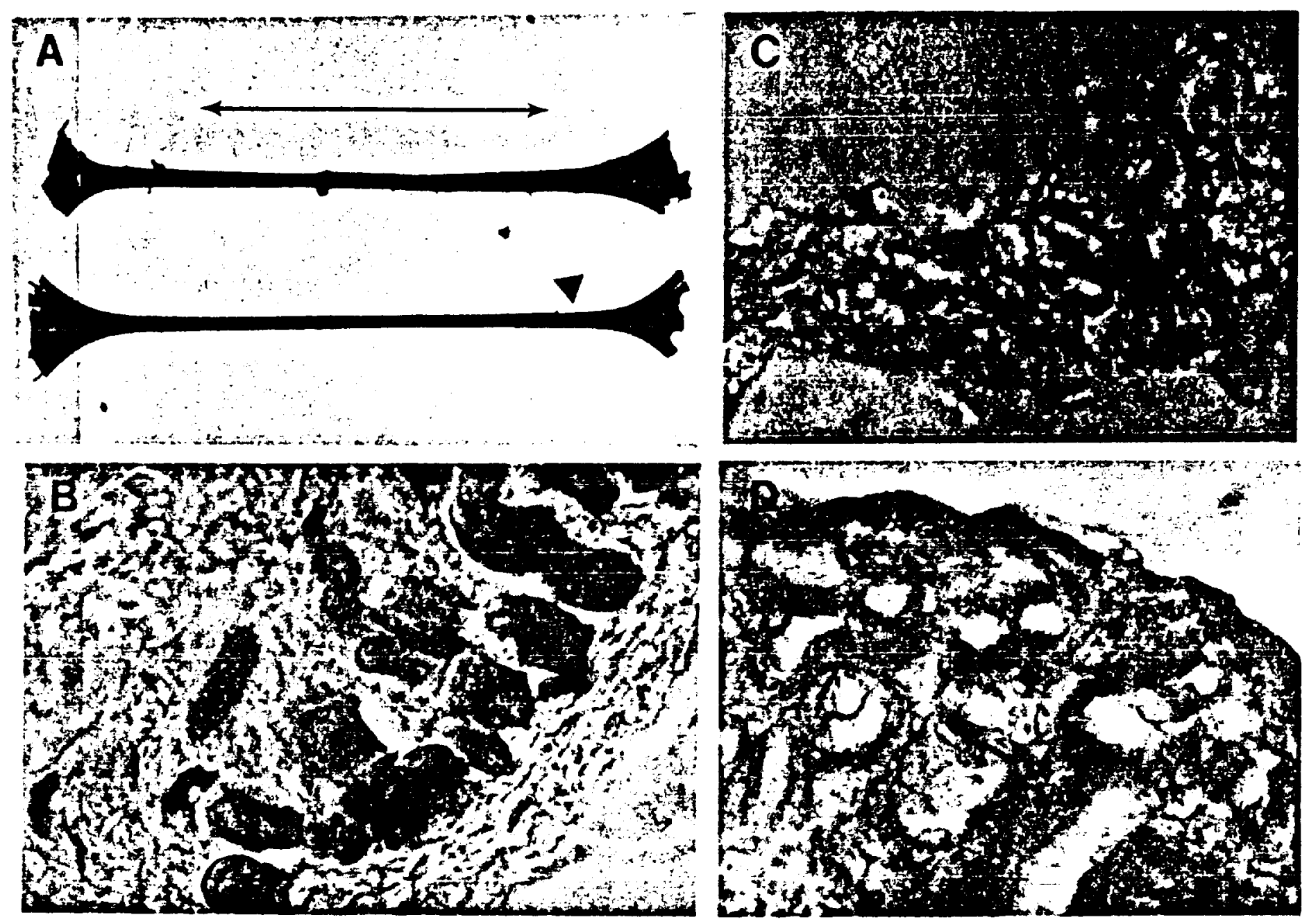

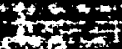

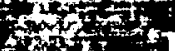

tots:

Vandenburgh et al. "Computer Aided Mechanogenesis of Skeletal Muscle organs from Single cells In Vitro"

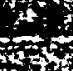
$+4+2$ Muscle Organ

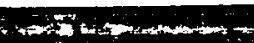

